International Journal of Pure and Applied Mathematics

Volume 104 No. 2 2015, 203-212

ISSN: 1311-8080 (printed version); ISSN: 1314-3395 (on-line version)

url: http://www.ijpam.eu

doi: http://dx.doi.org/10.12732/ijpam.v104i2.5

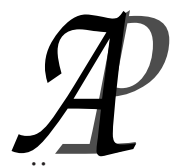

ijpam.eu

\title{
REVERSE DERIVATION ON SEMIRINGS
}

\author{
N. Sugantha Meena ${ }^{1}$, M. Chandramouleeswaran ${ }^{2} \S$ \\ ${ }^{1}$ Department of Mathematics \\ Kamaraj College of Engineering and Technology \\ Virudhunagar, INDIA \\ ${ }^{2}$ Department of Mathematics \\ SBK College \\ Aruppukottai, 626101, INDIA
}

\begin{abstract}
Motivated by some works on derivations on rings, Chandramouleeswaran and Thiruveni discussed the notion of derivations on semirings. In this paper, we introduce the notion of reverse derivations on semirings and prove some simple properties.
\end{abstract}

AMS Subject Classification: $16 \mathrm{Y} 30$

Key Words: reverse derivations, orthogonal reverse derivations

\section{Introduction}

The notion of derivations on rings is quite old, but got its significance only after Posner's work in [5]. Bresar and Vukuman [1] introduced the notion of orthogonal derivations on rings and the notion of reverse derivation in [2]. In [7], the authors discussed the notion of derivations and reverse derivations on semiprime rings.

Received: May 30, 2015

(C) 2015 Academic Publications, Ltd. url: www.acadpubl.eu

${ }^{\S}$ Correspondence author 
The notion of semirings was introduced by H.S.Vandiver [8] in the year 1934. In 2010, Chandramouleeswaran and Thiruveni introduced the theory of derivation on semirings [3]. They discussed in detail the notion of generalized derivations and $\alpha$ derivations in [4]. In our paper [6] we discussed the notion of orthogonal derivations on semirings. Motivated by these works, in this paper we introduced we introduce the notion of reverse derivations and orthogonal reverse derivations on semirings and discuss some elegant results.

\section{Preliminaries}

In this section, we recall some basic definitions and results that are needed for our work.

Definition 2.1. A semiring is a nonempty set $S$ on which operations of addition and multiplication have been defined such that

1. $(S,+)$ is a semigroup.

2. $(S, \cdot)$ is a semigroup.

3. Multiplication distributes over addition from either side.

Definition 2.2. Let $(S,+, \cdot)$ be a semiring. An element $a$ in $S$ is called additively left cancellative if $a+b=a+c \Longrightarrow b=c \forall b, c \in S$. It is said to be additively right cancellative if $b+a=c+a \Longrightarrow b=c$. The element $a \in S$ is said to be additively cancellative if it is both additively left and right cancellative. The Semiring $S$ is said to be additively cancellative if all the elements in $S$ are additively cancellative.

Definition 2.3. A semiring $(S,+, \cdot)$ is said to be additively commutative if $(S,+)$ is a commutative semigroup. A semiring $(S,+, \cdot)$ is said to be multiplicatively commutative if $(S, \cdot)$ is a commutative semigroup. It is said to be commutative if both $(S,+)$ and $(S, \cdot)$ are commutative.

Definition 2.4. Let $(S,+, \cdot)$ be a semiring. An element $a$ in $S$ is said to be multiplicatively idempotent if $a \cdot a=a$. If each element of $S$ is multiplicatively idempotent, then $S$ is said to be an multiplicatively idempotent semiring.

Definition 2.5. A Semiring $S$ is said to be Prime if $a S b=0 \Longrightarrow a=0$ or $b=0, a, b \in S$. A Semiring $S$ is said to be Semiprime if $a S a=0 \Longrightarrow a=$ $0, a \in S$. A Semiring $S$ is said to be 2-torsion free if $2 a=0 \Longrightarrow a=0, a \in S$. 
Definition 2.6. A Semiring $(S,+, \cdot)$ is said to be a semiring with zero, if it has an element 0 in $S$ such that $x+0=x=0+x$ and $x \cdot 0=0=0 \cdot x \quad \forall x \in S$. It is said to be a semiring with unity if there exists an element $1 \neq 0$ such that $x \cdot 1=x=1 \cdot x$

Definition 2.7. An additive mapping $d: S \rightarrow S$ is called a derivation on a semiring $S$ if $d(x y)=d(x) y+x d(y) \forall x, y \in S$.

Definition 2.8. Let $S$ be a semiring. Derivations $d$ and $g$ on $S$ are said to be orthogonal derivations on $S$ if $d(x) S g(y)=0=g(x) S d(y) \forall x, y \in S$.

Lemma 2.9. [6] Let $S$ be a 2 torsion free semiprime semiring, $a$ and $b$ the elements of $S$. Then the following are equivalent.

(i) $a S b=0$

(ii) $b S a=0$

(iii) $a S b+b S a=0$.

If one of above conditions is fulfilled then $a b=b a=0$

Lemma 2.10. [6] Let $S$ be a 2-torsion free semiprime semiring. Suppose that additive mappings $f$ and $g$ on a semiring $S$ satisfying $f(x) S g(x)=0, \forall x \in$ $S$ then $f(x) S g(y)=0 \forall x, y \in S$.

Lemma 2.11. [6] Let $S$ be a 2 torsion free semiprime semiring and let $d$ and $g$ be derivations on $S$. Then $d$ and $g$ are orthogonal iff $d(x) g(y)+g(x) d(y)=$ $0 \forall x, y \in S$.

Lemma 2.12. [6] Let $S$ be a 2 torsion free semiprime semiring. Suppose $d$ and $g$ are derivations on $S$. Then $d$ and $g$ are orthogonal iff $d g=0$.

Lemma 2.13. [6] Let $S$ be a 2 torsion free semiprime semiring.Suppose $d$ and $g$ are derivations on $S$. Then $d$ and $g$ are orthogonal iff $d g+g d=0$.

Lemma 2.14. [6] Let $S$ be a 2 torsionfree and additively cancellative semiprime semiring. Let $d$ and $g$ be derivations on $S$. Then $d$ and $g$ are orthogonal iff $d g$ is a derivation.

Notation. Throughout this paper we assume that $S$ is an additively commutative semiring with 0 and 1. 


\section{Reverse Derivation on Semirings}

In this section, we introduce the notion of reverse derivation on semirings. Examples are discused in detail to give a reverse derivation which is not a derivation. Some simple properties on reverse derivations are proved.

Definition 3.1. An additive map $d: S \rightarrow S$ is called a reverse derivation on $S$ if

$$
d(x y)=d(y) x+y d(x) \forall x, y \in S .
$$

Example 3.2. Let

$$
M_{2}(S)=\left\{\left(\begin{array}{cc}
a & 0 \\
b & a
\end{array}\right) \mid a, b \in S .\right\},
$$

where $S$ is a commutative semiring. Define $d: M_{2}(S) \rightarrow M_{2}(S)$ by

$$
d\left[\left(\begin{array}{ll}
a & 0 \\
b & a
\end{array}\right)\right]=\left(\begin{array}{ll}
0 & 0 \\
b & 0
\end{array}\right) .
$$

Then $d$ is both reverse derivation and derivation on $M_{2}(S)$.

Example 3.3. Let

$$
M_{2}(S)=\left\{\left(\begin{array}{ll}
a & 0 \\
b & c
\end{array}\right) \mid a, b, c \in S .\right\},
$$

where $S$ is any semiring. Define $d: M_{2}(S) \rightarrow M_{2}(S)$ by

$$
d\left[\left(\begin{array}{cc}
a & 0 \\
b & c
\end{array}\right)\right]=\left(\begin{array}{ll}
0 & 0 \\
b & 0
\end{array}\right) .
$$

Then $d$ is a derivation on $M_{2}(S)$ but not a reverse derivation on $S$.

Example 3.4. Let $S_{1}=S \bigoplus S$, where $S$ is any semiring. Let $d_{1}, d_{2}$ be any two reverse derivations on $S$. Let $D: S_{1} \rightarrow S_{1}$ defined by $D\left(x_{1}, x_{2}\right)=$ $\left(d_{2}\left(x_{1}\right), d_{1}\left(x_{2}\right)\right)$. Then $D$ is a reverse derivation but not a derivation on $S_{1}$.

Example 3.5. Let

$$
M_{2}(S)=\left\{\left(\begin{array}{cc}
a & b \\
0 & 0
\end{array}\right) \mid a, b \in S .\right\},
$$

where $S$ is any semiring. Define $d: M_{2}(S) \rightarrow M_{2}(S)$ by

$$
d\left[\left(\begin{array}{cc}
a & b \\
0 & 0
\end{array}\right)\right]=\left(\begin{array}{cc}
a & 0 \\
0 & 0
\end{array}\right) .
$$

Then $d$ is neither a reverse derivation nor a derivation on $M_{2}(S)$. 
Proposition 3.6. Let $d$ be a reverse derivation on a semiring $S$. If $S$ is of characteristic 2 then $d^{2}$ is a derivation.

Proof. We have

$$
\begin{aligned}
d^{2}(x y) & =d(d(x y)) \\
& =d(d(y) x+y d(x)) \\
& =d(x) d(y)+x d^{2}(y)+d^{2}(x) y+d(x) d(y) \\
& =d^{2}(x) y+x d^{2}(y)+2 d(x) d(y) \\
& \left.=d^{2}(x) y+x d^{2}(y) \text { (since } S \text { is of characteristic } 2\right)
\end{aligned}
$$

That is $d^{2}(x y)=d^{2}(x) y+x d^{2}(y)$. Therefore $d^{2}$ is a ususal derivation.

Proposition 3.7. If $e$ is a multiplicative idempotent element of an additively cancellative semiring.then $e d(e) e=0$.

Proof. Since $e$ is an multiplicaive idempotent $e \cdot e=e$. Then $d(e \cdot e)=d(e)$.

Since $d$ is a reverse derivation we have $d(e) e+e d(e)=d(e)$. Premultiplying by $e$ we have $e d(e) e+e . e d(e)=e d(e)$. That is $e d(e) e+e d(e)=e d(e)+0$. Since $S$ is additively cancellative we have $e d(e) e=0$

Proposition 3.8. If $e$ is a commuting idempotent then $d(e)=0$

Proof. Since $e$ is a commuting idompotent $a \cdot e=e \cdot a$.

In particular $e d(e)=d(e) e$. Postmultiplying by $e$ we get $e d(e) e=d(e) e \cdot e=$ $d(e) e$. By proposition 3.7 we have $d(e) \cdot e=0$

$$
\begin{aligned}
d(e) & =d(e . e)=d(e) e+e d(e) \\
& =d(e) e+d(e) e=0
\end{aligned}
$$

That is $d(e)=0$.

Corollary 3.9. If $1 \in S$ then $d(1)=0$

Proposition 3.10. If $d$ is a reverse derivation then

$$
\begin{aligned}
d^{n}(x y) & =\sum_{r=0}^{n} n C r d^{(n-r)}(y) d^{r}(x) \text { if } n \text { is odd } \\
& =\sum_{r=0}^{n} n C r d^{(n-r)}(x) d^{r}(y) \text { if } n \text { is even }
\end{aligned}
$$

Proof. we prove the result using induction on $n$. 
The result is obviously true for $\mathrm{n}=1$ Assume $n=2$

$$
\begin{aligned}
d^{2}(x y) & =d^{2}(x) y+2 d(x) d(y)+x d^{2}(y) \\
& =2 C_{0} d^{2-0}(x) d^{0}(y)+2 C_{1} d^{2-1}(x) d^{1}(y)+2 C_{2} d^{2-2}(x) d^{2}(y) \\
& =\sum_{r=0}^{2} 2 C_{r} d^{(2-r)}(x) d^{r}(y)
\end{aligned}
$$

The result is true for $n=2$.

Assume the result is true for $2 n-1$ and $2 n$. we use the following identities $n C_{r}=n C_{n-r}$ and $(n-1) C_{r}+(n-1) C_{r-1}=n C_{r}$. We shall prove the result for $2 n+1$ case and the proof for the even $n$ will be analogous.

$$
\begin{aligned}
& d^{2 n+1}(x y)=d\left(d^{2 n}(x y)\right) \\
= & d\left(\sum_{r=0}^{2 n} 2 n C_{r} d^{2 n-r}(x) d^{r}(y)\right) \\
& =\sum_{r=0}^{2 n} 2 n C_{r} d\left(d^{2 n-r}(x) d^{r}(y)\right) \\
= & \left.\sum_{r=0}^{2 n} 2 n C_{r}\left[d^{r+1}(y) d^{2 n-r}(x)\right)+d^{r}(y) d^{2 n-r+1}(x)\right] \\
= & \left.\sum_{r=0}^{2 n} 2 n C_{r}\left[d^{r+1}(y) d^{2 n-r}(x)\right]+\sum_{r=0}^{2 n} 2 r d^{r}(y) d^{2 n-r+1}(x)\right] \\
= & d(y) d^{2 n}(x)+2 n C_{1} d^{2}(y) d^{2 n-1}(x)+\ldots \\
& +2 n C_{i-1} d^{i}(y) d^{2 n-(i-1)}(x)+2 n C_{i} d^{i+1}(y) d^{2 n-i}(x)+\ldots \\
& +2 n C_{2 n-1} d^{2 n}(y) d(x)+d^{2 n+1}(y) x \\
& +y d^{2 n+1}(x)+2 n C_{1} d(y) d^{2 n}(x)+\ldots \\
& +2 n C_{i-1} d^{i-1}(y) d^{2 n-i}(x)+2 n C_{i} d^{i}(y) d^{2 n-i+1}(x)+\ldots \\
& +2 n C_{2 n-1} d^{2 n-1}(y) d^{2}(x)+d^{2 n}(y) d(x) \\
= & d^{2 n+1}(y) x+(2 n+1) C_{1} d^{2 n}(y) d(x)+\ldots+ \\
& (2 n+1) C_{i} d^{2 n-i+1}(y) d(x)+\ldots+(2 n+1) C_{2 n} d(y) d^{2 n}(x) \\
& +y d^{2 n+1}(x) \\
& \sum_{r=0} n C_{r} d^{(2 n+1-r)}(y) d^{r}(x) \\
& \\
& \\
& \\
&
\end{aligned}
$$


Proposition 3.11. If $d$ is a reverse derivation then for any $n \geq 2$

$$
d\left(x^{n}\right)=\sum_{r=0}^{n-1} x^{r} d(x) x^{(n-1)-r}
$$

We prove the result using induction on $n$. For $n=2$

$$
\begin{aligned}
d\left(x^{2}\right) & =d(x \cdot x)=d(x) x+x d(x) \\
& =x^{0} d(x) x^{(2-1)-0}+x^{1} d(x) x^{(2-1)-1} \\
& =\sum_{r=0}^{2-1} x^{r} d(x) x^{(2-1)-r}
\end{aligned}
$$

Assume the result is true of $n-1$, and prove for any $n$.

$$
\begin{aligned}
d\left(x^{n}\right) & =d\left(x^{n-1} \cdot x\right) \\
& =d(x) x^{n-1}+x d\left(x^{n-1}\right) \\
& =x^{0} d(x) x^{(n-1)-0}+x \sum_{r=0}^{n-2} x^{r} d(x) x^{(n-2)-r} \\
& =x^{0} d(x) x^{(n-1)-0}+\sum_{r=0}^{n-2} x^{r+1} d(x) x^{(n-2)-r} \\
& =x^{0} d(x) x^{(n-1)}+x^{1} d(x) x^{(n-2)}+x^{2} d(x) x^{(n-3)}+\ldots+x^{(n-1)} d(x) x^{0} \\
& =\sum_{r=0}^{n-1} x^{r} d(x) x^{(n-1)-r}
\end{aligned}
$$

\section{Orthogonal Reverse Derivations on Semirings}

In this section we introduce the notion of orthogonal reverse derivations on semirings and prove some theorems.

Definition 4.1. Let $S$ be a semiring. The reverse derivations $d$ and $g$ on $S$ are said to be orthogonal on $S$ if $d(x) S g(y)=0=g(x) S d(y) \forall x, y \in S$.

Example 4.2. Let $S_{1}$ and $S_{2}$ be two semirings and let $S=S_{1} \oplus S_{2}$. Let $d_{1}$ be a non zero reverse derivation of $S_{1}$. A mapping $d: S \rightarrow S$ defined by $d\left(s_{1}, s_{2}\right)=\left(d_{1}\left(s_{1}\right), 0\right)$ is a non zero reverse derivation of $S$. We write $d$ as $d_{1} \oplus 0$. Let $d_{2}$ be a non zero reverse derivation of $S_{2}$. A mapping $g: S \rightarrow S$ defined by $g\left(s_{1}, s_{2}\right)=\left(0, d_{2}\left(s_{2}\right)\right)$, write $g$ as $0 \oplus d_{2}$. Then $d$ and $g$ are orthogonal. 
Analogous to Lemma 2.12, 2.13, 2.14, 2.15 we can prove the following results.

Lemma 4.3. Let $S$ be a 2-torsion free semiprime semiring and let $d$ and $g$ be reverse derivations on $S$. Derivations $d, g$ are orthogonal iff $d(x) g(y)+$ $g(x) d(y)=0 \forall x, y \in S$.

Theorem 4.4. Let $S$ be a 2 torsion free semiprime semiring. Let $d$ and $g$ be reverse derivations on $S$. Then $d$ and $g$ are orthogonal if and only if $d g=0$

Theorem 4.5. Let $S$ be a 2 torsion free semiprime semiring. Let $d$ and $g$ be reverse derivation on $S$. Then $d$ and $g$ are orthogonal iff $d g+g d=0$.

Theorem 4.6. Let $S$ be a 2 - torsion free and additively cancellative semiprime semiring. Let $d$ and $g$ be reverse derivation on $S$. Then $d$ and $g$ are orthogonal iff $d g$ is a derivation on $S$.

Theorem 4.7. Let $S$ be a 2-torsion free prime semiring. Suppose that $d$ and $g$ are reverse derivations on $S$ such that $d g$ is a derivation. Then either $d=0$ or $g=0$.

Proof. Suppose that $d$ and $g$ are reverse derivations on $S$, such that $d g$ is a derivation. By Theorem $4.6 d$ and $g$ are orthogonal derivations on $S$, so $d(x) S g(y)=0=g(x) S d(y)$. In Particulard $(x) S g(x)=0$. Since $S$ is prime semiring either $d(x)=0$ or $g(x)=0, \forall x \in S$.

That is either $d=0$ or $g=0$.

Theorem 4.8. Let $S$ be a 2 torsionfree semiprime semiring. Let $d$ and $g$ be reverse derivations on $S$.Then $d$ and $g$ are orthogonal iff $d(x) g(x)=0$.

Proof. Suppose $d(x) g(x)=0$.

Replacing $x$ by $x+y$ and simplifying we have $d(x) g(y)+d(y) g(x)=0$.

Replacing $y$ by $y x$ and simplifying we get $d(x) x g(y)+d(x) y g(x)+x d(y) g(x)=$ 0.

Replacing $y$ by $x$ we have $d(x) x g(x)+d(x) x g(x)+x d(x) g(x)=0$.

That is $2 d(x) x g(x)=0$. Since $S$ is 2 torsion free we have $d(x) x g(x)=$ $0, \forall x \in S$.

That is $d(x) S g(x)=0$. By lemma $2.10 d(x) S g(y)=0$. By lemma 2.9 $g(y) S d(x)=0$. That is $g(x) S d(y)=0$. Therefore $d$ and $g$ are orthogonal.

Conversely assume $d$ and $g$ are orthogonal. That is $d(x) S g(y)=0$. In Particular $d(x) S g(x)=0$. By lemma 2.9 we have $d(x) g(x)=0$.

Corollary 4.9. Let $S$ be a 2-torsion free semiprime semiring and let $d$ be a reverse derivation on $S$. If $d(x)^{2}=0, \forall x \in S$, then $d=0$. 
Proof. $d(x) d(x)=0$. By above theorem $d$ and $d$ are orthogonal on $S$, so $d(x) S d(y)=0$. In particular $d(x) S d(x)=0$.

Since $S$ is semiprime $d(x)=0, \forall x \in S$. Thus $d=0$.

Corollary 4.10. Let $S$ be a 2 torsionfree and additively cancellative semiprime semiring and $d$ be a reverse derivation on $S$. If $d^{2}$ is a derivation then $d=0$.

Proof. We have $d^{2}(x y)=d(d x y)=x d^{2}(y)+d^{2}(x) y+2 d(x) d(y)$.

But $d^{2}(x y)=d^{2}(x) y+x d^{2}(y)$.

Since $S$ is 2-torsion free and additively cancellative $d(x) d(y)=0$.

Replacing $y$ by $x y$ and simplifying we get $d(x) y d(x)=0, \forall x, y \in S$. $d(x) S d(x)=0$. Since $S$ is semiprime $d(x)=0, \forall x \in S$. That is $d=0$.

Remark 4.11. From the above corollary we infer that a non zero derivation cannot be orthogonal on itself.

\section{References}

[1] M. Bresar, J. Vukuman, Orthogonal derivation and extension of a theorem of Posner, Radovi Matematicki, 5 (1989), 237-246.

[2] M. Bresar, J. Vukuman, On some additive mappipngs in rings with involution, Aequations Math., 38 (1989), 178-185.

[3] M. Chandramouleeswaran, V. Thiruveni, On derivations of semirings, Advances in Algebra, 3 (2010), 123-131.

[4] M. Chandramouleeswaran, V. Thiruveni, A note on $\alpha$ derivations in semirings, International Journal of Pure and Applied Science and Technology (2011), 71-77.

[5] E.C. Posner, Derivation in prime rings, American Math. Soc., 8 (1957), 1093-1100.

[6] N. Sugantha Meena, M. Chandramouleeswaran, Orthogonal derivations on semirings, International Journal of Contemporary Mathematical Sciences, 9, No. 13 (2014), 645-651.

[7] Samman Mohammed, Nouf Alyamani, Derivations and reverse derivations on semiprime rings, International Mathematical Forum, 2, No. 39 (2007), 1895-1902. 
[8] H.S. Vandiver, Note on a simple type of algebra in which the cancellation law of addition does not hold, Bull. Amer. Math. Soc., 40 (1934), 916-920. 